\title{
Facile and efficient two-step formation of renewable monomer 2,5-furandicarboxylic acid from carbohydrates over NiOx catalyst
}

\author{
Huai Liu, ${ }^{a}$ Weile Li, ${ }^{a}$ Miao Zuo, ${ }^{a}$ Xing Tang, ${ }^{* a, b}$ Xianhai Zeng,,${ }^{a, b}$ Yong Sun,,${ }^{a, b}$ \\ Tingzhou Lei, ${ }^{\mathrm{c}}$ Huayu Fang, ${ }^{\mathrm{d}}$ Tianyuan $\mathrm{Li}^{\mathrm{d}}$ and Lu Lin*a,b
}

a Xiamen Key Laboratory of Clean and High-valued Applications of Biomass, College of Energy,

Xiamen University, Xiang'an South Road, Xiamen 361102, China.

${ }^{\mathrm{b}}$ Fujian Engineering and Research Center of Clean and High-valued Technologies for Biomass,

Xiamen University, Xiang'an South Road, Xiamen 361005, Fujian, China

${ }^{\mathrm{c}}$ Henan Key Lab of Biomass Energy, Huayuan Road 29, Zhengzhou 450008, Henan, China

${ }^{\mathrm{d}}$ Fujian Huafeng New Materials Co., Ltd., Putian 351152, Fujian, China.

*Corresponding authors. E-mail addresses: x.tang@xmu.edu.cn (X. Tang); lulin@xmu.edu.cn (L. Lin).

Number of pages: 15

Number of figures: 10 
Number of tables: 4
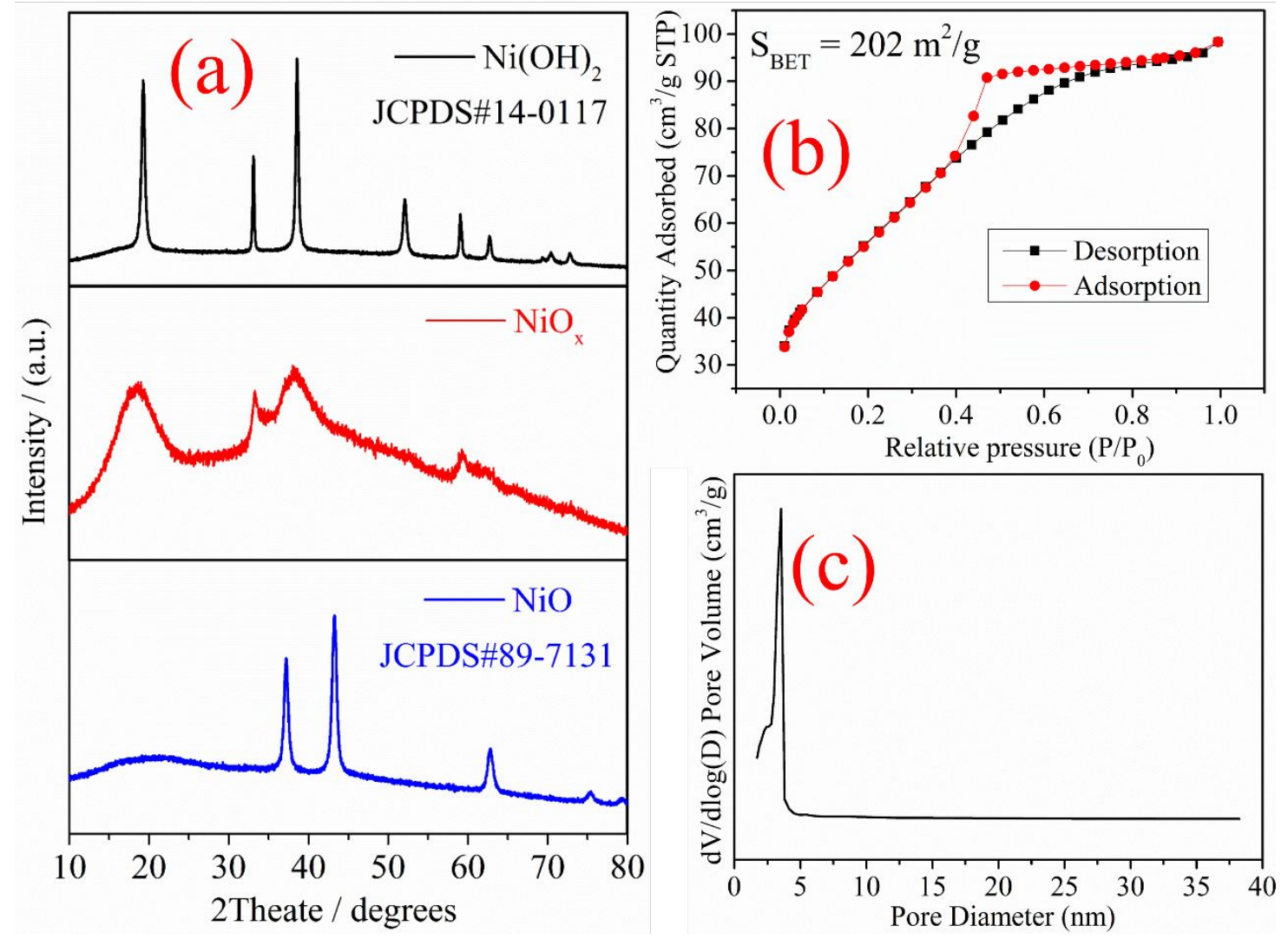

Figure S1. The XRD patterns of the synthesized catalysts (a), $\mathrm{N}_{2}$ adsorption/desorption isotherm of $\mathrm{NiO}_{\mathrm{x}}(\mathrm{b})$, pore size distribution curves of $\mathrm{NiO}_{\mathrm{x}}(\mathrm{c})$. 

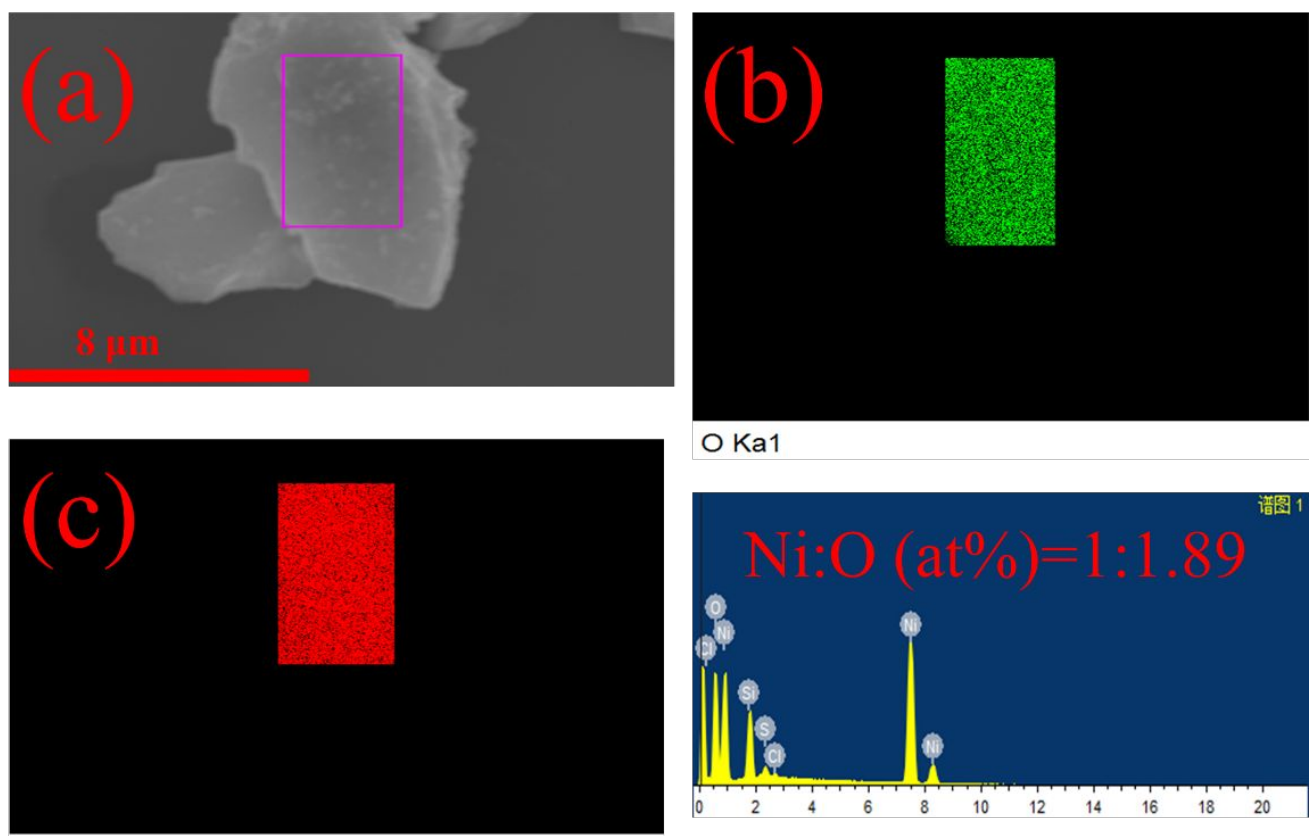

Ni Ka1

Figure S2. SEM image (a) and EDX elemental maps of the prepared $\mathrm{NiO}_{\mathrm{x}}(\mathrm{b}) \mathrm{O}$, (c) Ni. 


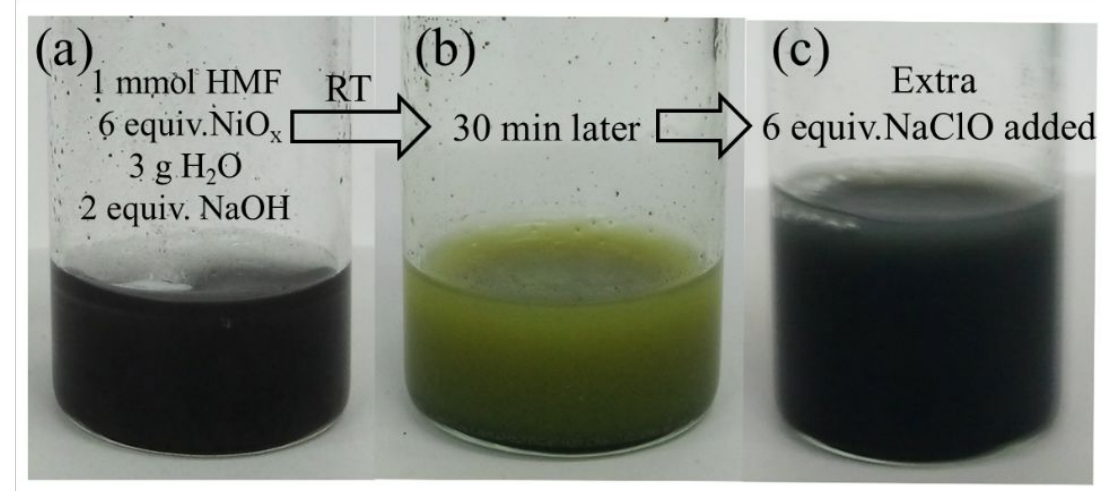

Figure S3. The photos of the sample in Table 1, entry 2 before (a) and after (b) reaction. The photo (c) was taken after 6 equivalents of $\mathrm{NaClO}$ were added into (b) mixtures. An FDCA yield of $85 \%$ and full HMF conversion were detected after 10 min reaction of the addition of 6 equivalents of $\mathrm{NaClO}$. 


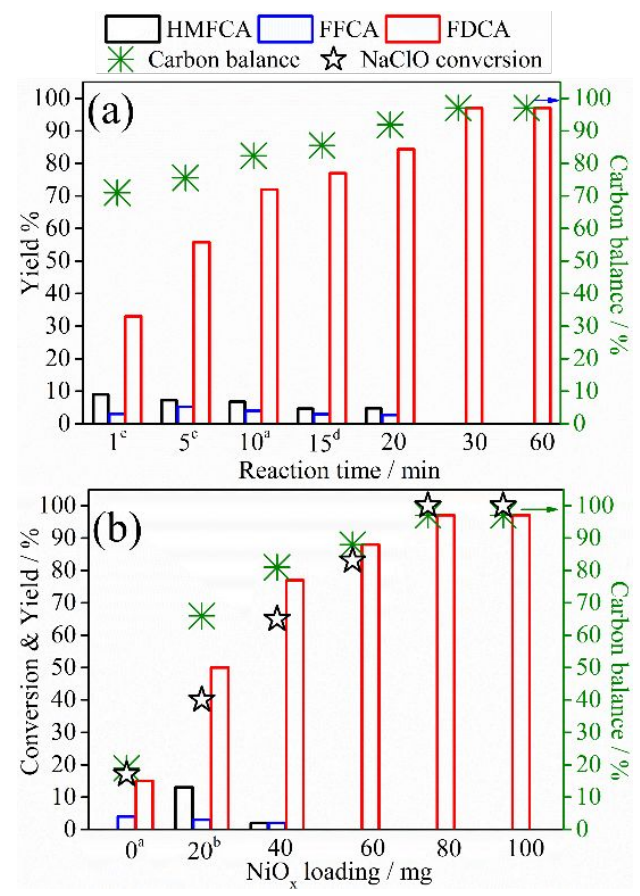

Figure S4. Effect of $\mathrm{NiO}_{\mathrm{x}}$ loading (a) and reaction time (b). If not specified, the default reaction conditions are as follows: $\mathrm{HMF}(1 \mathrm{mmol}), \mathrm{NiO}_{\mathrm{x}}(80 \mathrm{mg}), 7.5 \mathrm{wt} \% \mathrm{NaClO}$ aqueous solution $(6 \mathrm{mmol} \mathrm{NaClO}), 25^{\circ} \mathrm{C}, 30 \mathrm{~min}$. The $\mathrm{HMF}$ conversion was $100 \%$ except in the marked samples. ( ${ }^{\text {a }} 96 \%,{ }^{\text {b }} 98 \%,{ }^{\text {c }} 90 \%,{ }^{d} 99 \%,{ }^{\text {e }} 63 \%$ ). 
Table S1. The effect of the type of the oxidant.

\begin{tabular}{ccccc}
\hline Entry & Oxidant & $\begin{array}{c}\text { HMF } \\
\text { conversion } \\
(\%)\end{array}$ & $\begin{array}{c}\text { FFCA yield } \\
(\%)\end{array}$ & $\begin{array}{c}\text { FDCA yield } \\
(\%)\end{array}$ \\
\hline $1^{\mathrm{a}}$ & $1 \mathrm{MPa} \mathrm{O}_{2}$ & 65 & 2 & 3 \\
$2^{\mathrm{b}}$ & $6 \mathrm{mmol} \mathrm{\textrm {H } _ { 2 }}$ & 26 & 4 & 2 \\
$3^{\mathrm{b}}$ & $6 \mathrm{mmol} \mathrm{TBHP}$ & 15 & 3 & 0 \\
4 & $6 \mathrm{mmol} \mathrm{NaClO}$ & 100 & 0 & 97 \\
$5^{\mathrm{b}}$ & $3 \mathrm{mmol} \mathrm{NaClO}_{2}$ & 13 & 2 & 0 \\
$6^{\mathrm{b}}$ & $1.5 \mathrm{mmol} \mathrm{NaClO}_{4}$ & 23 & 5 & 0 \\
\hline
\end{tabular}

Reaction condition: $\mathrm{HMF}(1 \mathrm{mmol})$, oxidant, $\mathrm{NiO}_{2}(80 \mathrm{mg}), 25{ }^{\circ} \mathrm{C}, 30 \mathrm{~min} .{ }^{\mathrm{a}} \mathrm{NaHCO}_{3}$ (2 mmol), $\mathrm{H}_{2} \mathrm{O}(3 \mathrm{~g}), 100{ }^{\circ} \mathrm{C}, 2 \mathrm{~h} .{ }^{\mathrm{b}} \mathrm{NaHCO}_{3}(2 \mathrm{mmol}), \mathrm{H}_{2} \mathrm{O}(3 \mathrm{~g})$. 
Table S2. The effect of the amount of $\mathrm{NaClO}$.

\begin{tabular}{cccccccc}
\hline Entry & $\begin{array}{c}\mathrm{NaClO} \\
(\mathrm{mmol})\end{array}$ & $\begin{array}{c}\text { NaClO } \\
\text { Conversion } \\
(\%)\end{array}$ & $\begin{array}{c}\text { HMF } \\
\text { conversion } \\
(\%)\end{array}$ & $\begin{array}{c}\text { HMFCA } \\
\text { yield } \\
(\%)\end{array}$ & $\begin{array}{c}\text { FFCA } \\
\text { yield } \\
(\%)\end{array}$ & $\begin{array}{c}\text { FDCA } \\
\text { yield } \\
(\%)\end{array}$ & $\begin{array}{c}\text { Carbon } \\
\text { balance } \\
(\%)\end{array}$ \\
\hline 1 & 3 & 99 & 84 & 20 & 6 & 43 & 82 \\
2 & 4 & 99 & 94 & 15 & 6 & 65 & 92 \\
3 & 6 & 98 & 100 & 0 & 0 & 97 & 97 \\
\hline
\end{tabular}

Reaction condition: $\mathrm{HMF}(1 \mathrm{mmol}), \mathrm{NiO}_{\mathrm{x}}(80 \mathrm{mg}), 7.5 \mathrm{wt} \% \mathrm{NaClO}$ aqueous solution, $25^{\circ} \mathrm{C}$, $30 \mathrm{~min}$. 
Table S3. Comparison of $\mathrm{NiO}_{\mathrm{x}}$ catalytic system with the recently published works for the production of FDCA.

\begin{tabular}{|c|c|c|c|c|c|c|}
\hline Entry & $\begin{array}{c}\text { Reaction } \\
\text { Temperatur } \\
\mathrm{e} \\
\left({ }^{\circ} \mathrm{C}\right) \\
\end{array}$ & $\begin{array}{l}\text { Reaction } \\
\text { Time }\end{array}$ & $\begin{array}{c}\text { Substrate } \\
\text { Concentration } \\
(w t \%)\end{array}$ & $\begin{array}{l}\text { FDCA } \\
\text { yield }\end{array}$ & E factor ${ }^{\mathrm{a}}$ & Ref. \\
\hline 1 & 120 & $16 \mathrm{~h}$ & 0.6 & 97 & 132 & $\begin{array}{c}\text { ChemSusChem } 11.13 \text { (2018): 2083- } \\
2090 .\end{array}$ \\
\hline 2 & 130 & $4 \mathrm{~h}$ & 0.19 & 99 & 432 & $\begin{array}{l}\text { Green chem. } 20.17 \text { (2018): } \\
\text { 3921-3926. }\end{array}$ \\
\hline 3 & 85 & $10 \mathrm{~h}$ & 0.6 & 93 & 138 & $\begin{array}{c}\text { Appl. Catal., B, } 244 \text { (2019): } \\
\text { 965-973. }\end{array}$ \\
\hline 4 & 90 & $6 \mathrm{~h}$ & 0.25 & 70 & 458 & $\begin{array}{c}\text { Green Chem. (2019). DOI: } \\
\text { 10.1039/c9gc01283d }\end{array}$ \\
\hline 5 & 25 & $30 \mathrm{~min}$ & 4.2 & 97 & 20 & This work \\
\hline
\end{tabular}

${ }^{\mathrm{a}} \mathrm{E}$ factor $=\frac{\text { Total waste }(\mathrm{Kg})}{\text { Product }(\mathrm{Kg})}$ 


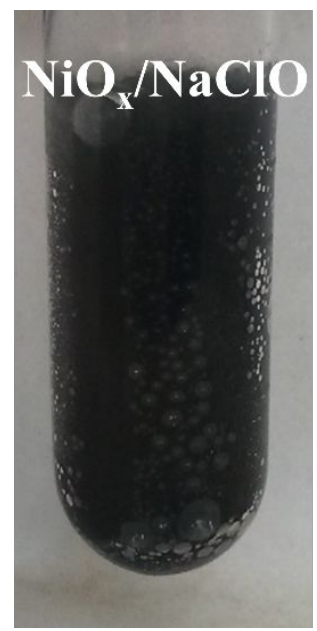

Figure S5. The photo of $\mathrm{NiO}_{\mathrm{x}}$ and $\mathrm{NaClO}$ mixtures. The photo was taken when adding $\mathrm{NiO}_{\mathrm{x}}(80 \mathrm{mg})$ to $7.5 \mathrm{wt} \% \mathrm{NaClO}$ aqueous solution $(3 \mathrm{~g}) 1$ minute later. The conversion of $\mathrm{NaClO}$ was $58 \%$ after 30 minutes agitation. 


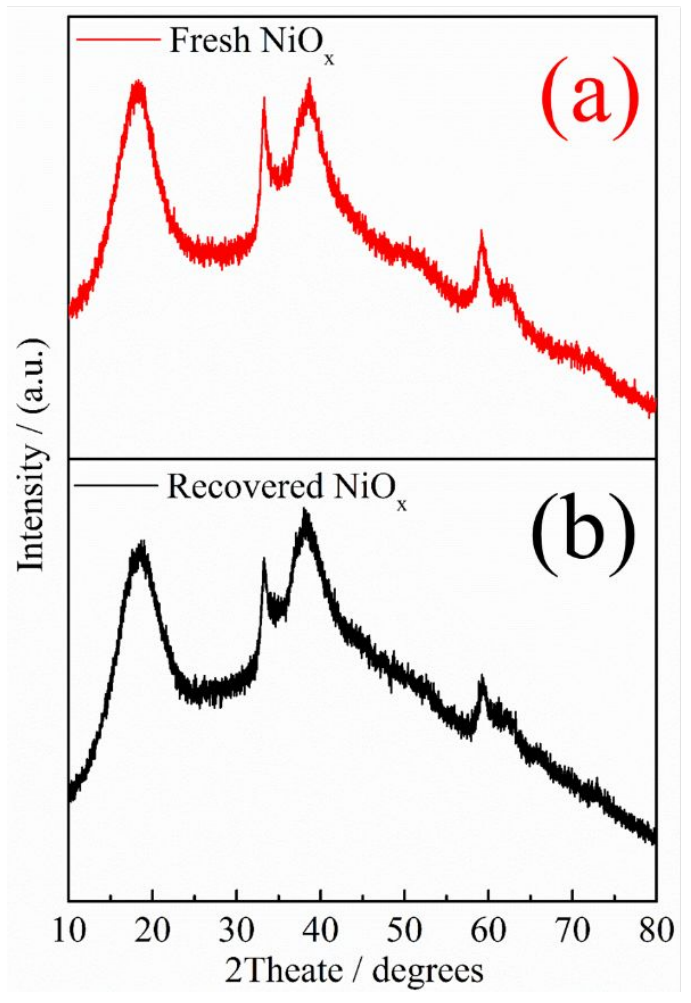

Figure S6. XRD patterns of (a) fresh $\mathrm{NiO}_{\mathrm{x}}$ and (b) $\mathrm{NiO}_{\mathrm{x}}$ reused after eleven runs for the oxidation of pure HMF. 


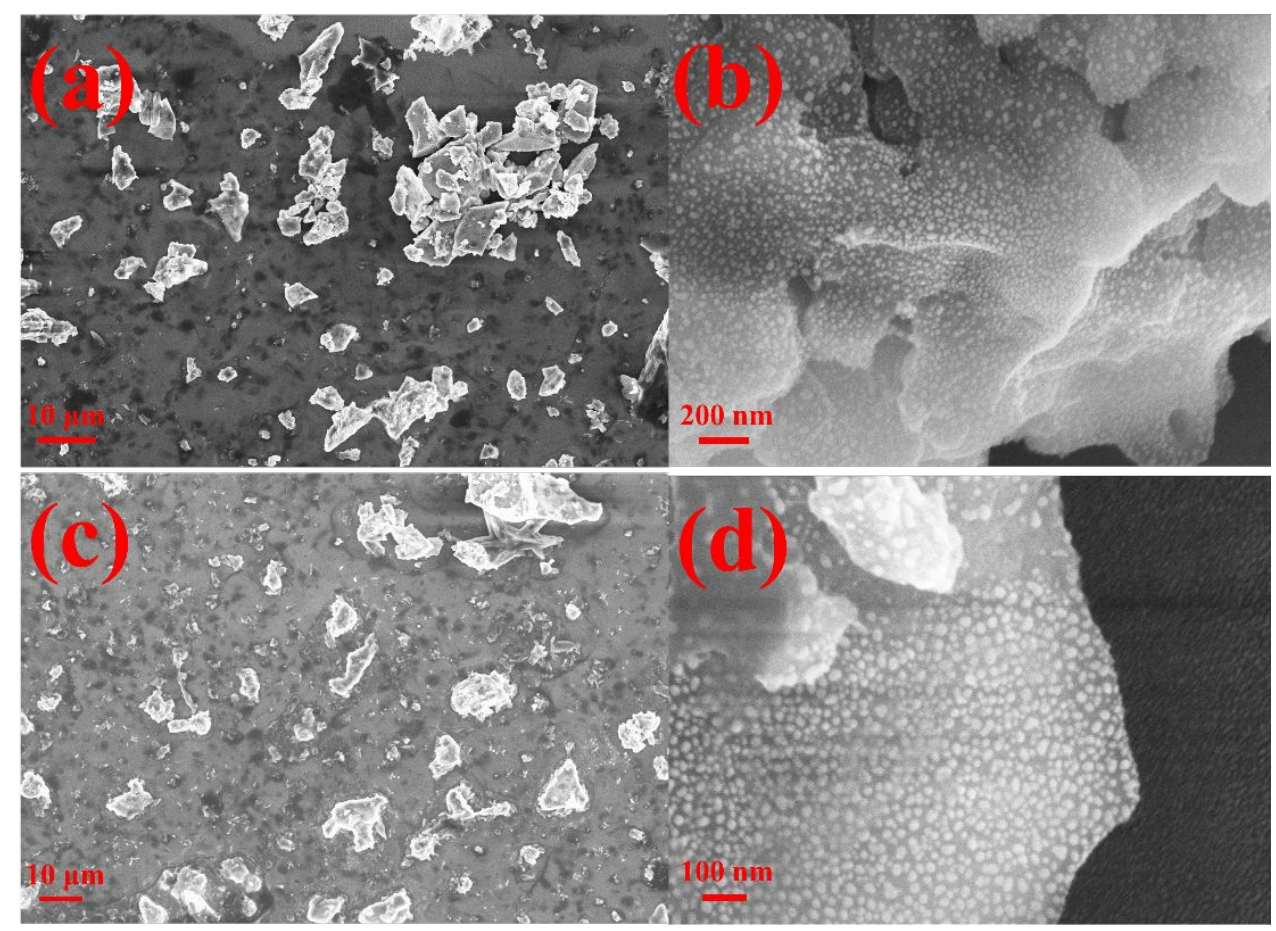

Figure S7. SEM images of (a), (b) fresh $\mathrm{NiO}_{\mathrm{x}}$ and (c), (d) $\mathrm{NiO}_{\mathrm{x}}$ reused after eleven runs for the oxidation of pure HMF. 


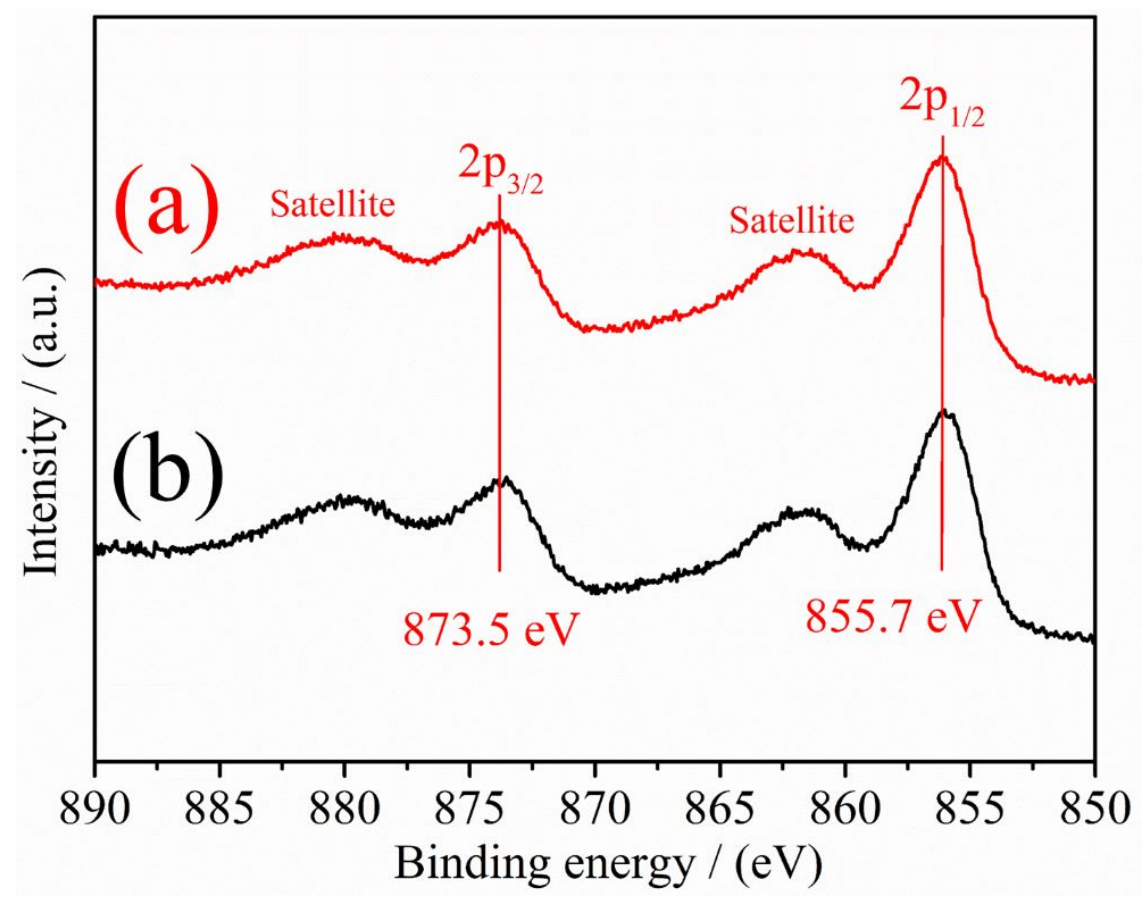

Figure S8. High-resolution XPS spectra $\mathrm{Ni} 2 \mathrm{p}$ of fresh $\mathrm{NiO}_{\mathrm{x}}(\mathrm{a})$ and $\mathrm{NiO}_{\mathrm{x}}$ reused after eleven runs (b) for the oxidation of pure HMF. 
$\underline{\text { Table S4. Surface area and carbon deposit of fresh } \mathrm{NiO}_{\mathrm{x}} \text { and reused } \mathrm{NiO}_{\mathrm{x}}}$.

\begin{tabular}{|c|c|c|c|}
\hline Enry & Material & $\begin{array}{l}\text { Surface area } \\
\qquad\left(\mathrm{m}^{2} / \mathrm{g}\right)\end{array}$ & $\begin{array}{l}\text { Carbon deposit } \\
\qquad(\mathrm{wt} \%)^{\mathrm{c}}\end{array}$ \\
\hline 1 & $\mathrm{NiO}_{\mathrm{x}}$-fresh & 202 & 0.00 \\
\hline 2 & $\begin{array}{l}\mathrm{NiO}_{\mathrm{x}} \text { - reused after } \\
\text { eleven runs }\end{array}$ & 198 & 0.34 \\
\hline 3 & $\begin{array}{c}\mathrm{NiO}_{\mathrm{x}}-\text { reused after } \\
\text { three runs }{ }^{\mathrm{b}}\end{array}$ & 168 & 3.30 \\
\hline 4 & $\begin{array}{c}\text { Refreshed } \mathrm{NiO}_{x} \text { by } \\
\mathrm{NaClO}\end{array}$ & 197 & 0.68 \\
\hline
\end{tabular}

${ }^{a}$ The substrate is pure HMF. ${ }^{b}$ The substrate is glucose derived crude HMF. ${ }^{c}$ Determined by an Elementar Vario EL III (Germany). 


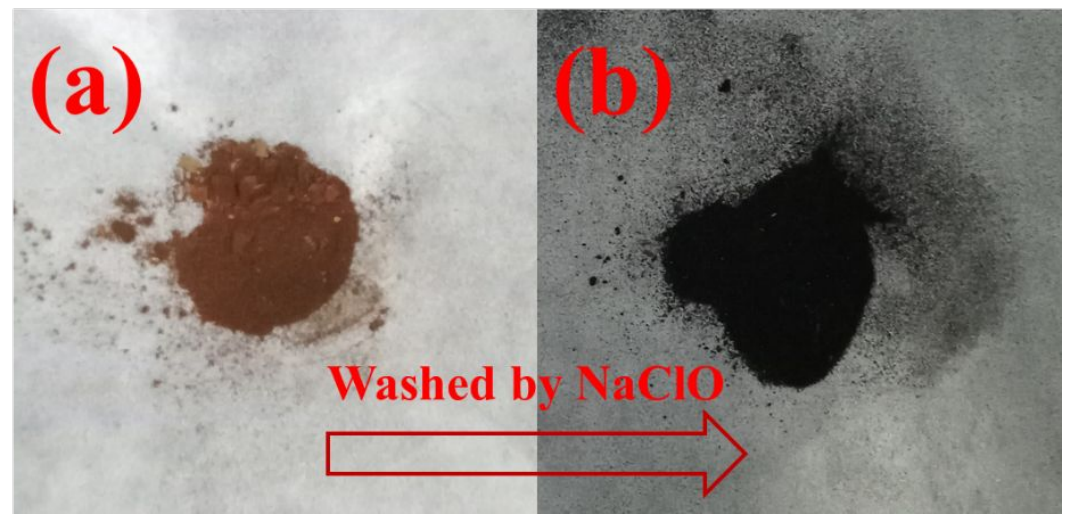

Figure S9. The $\mathrm{NiO}_{\mathrm{x}}$ recovered from the reusability test of the oxidation of glucosederived crude $\mathrm{HMF}$ in third runs (a), the regenerated $\mathrm{NiO}_{\mathrm{x}}$ by washing with $10 \mathrm{~mL} 7.5$ $w t \% \mathrm{NaClO}$ aqueous solution for 30 minutes (b). 


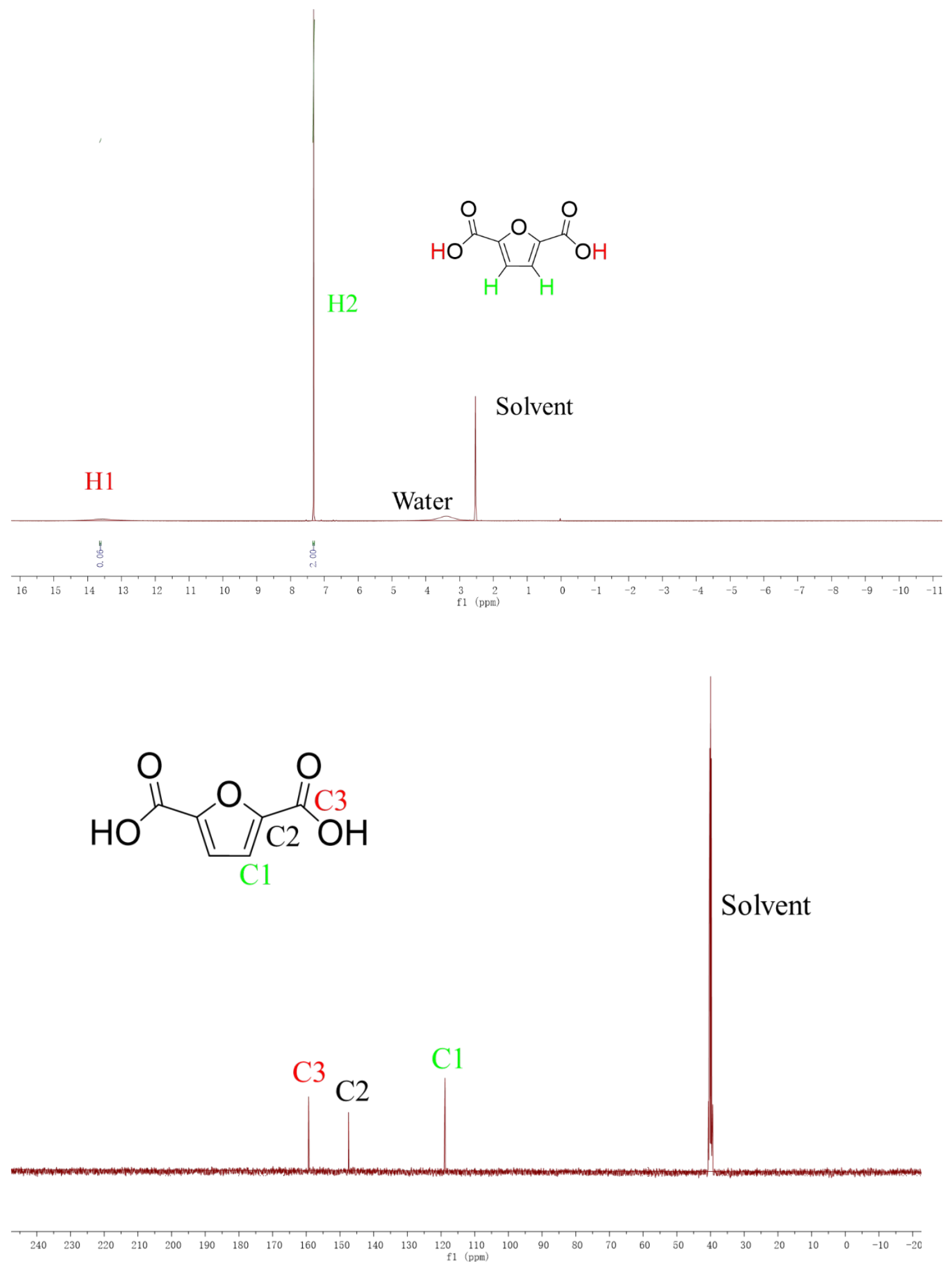

Figure S10. The ${ }^{1} \mathrm{HNMR}$ and ${ }^{13} \mathrm{CNMR}$ spectrum of the obtained FDCA. 